\title{
ANALISIS DE LA EVALUACION MAS RECIENTE DEL ESTADO DE CONSERVACION DE LOS ANFIBIOS Y REPTILES DE ARGENTINA
}

\author{
ANALYSIS OF THE LAST ASSESSMENT OF CONSERVATION STATUS OF \\ AMPHIBIANS AND REPTILES FROM ARGENTINA
}

\author{
Carmen Ubeda \& Dora Grigera \\ Centro Regional Bariloche, Universidad Nacional del Comahue, Unidad Postal Universidad, R 8400 FRF - San \\ Carlos de Bariloche, Prov. de Río Negro, Argentina. E-mail: cubeda@ bariloche.com.ar, \\ dgrigera@crub.uncoma.edu.ar
}

\begin{abstract}
RESUMEN
Sobre la base de una evaluación del estado de conservación de la herpetofauna de Argentina, publicada recientemente, se analizan los factores determinantes del estatus asignado, con énfasis en las especies de las categorías que indican una mayor amenaza. De los 484 taxa evaluados el $23 \%$ tiene problemas de conservación. Una proporción importante de estas especies tiene áreas de distribución restringidas, especialización en el uso del hábitat, está poco protegida y sufre acciones extractivas. Se analizan los principales factores de amenaza sobre los herpetozoos, encontrándose que la mayoría está afectada por la alteración del hábitat. Los reptiles sufren el comercio más intensamente que los anfibios. Se comparan los resultados de la nueva evaluación con la previa. El número de taxa de anfibios categorizados se incrementó un $42 \%$ y el de reptiles un $58 \%$. Aparece la categoría Insuficientemente Conocida, que comprende el $33 \%$ de la herpetofauna.
\end{abstract}

Palabras claves: estado de conservación, amenazas, anfibios, reptiles, Argentina.

ABSTRACT

Based on the new evaluation of the conservation status of Argentinian herpetofauna, recently published, the factors determining the assigned status are analyzed, with emphasis on the threatened species. Of the 484 taxa evaluated, $23 \%$ has conservation problems. An important proportion of these species have restricted distribution areas, are habitat specialists, have poor protection and are subject to extractive actions. The main factors of threat to the herpetozoos are analyzed. The majority of them is affected by the alteration of the habitat. Reptiles undergo the trade more intensely than amphibians. The results of the new evaluation and the previous one are compared. The number of taxa of categorized amphibians was increased by $42 \%$ and that of reptiles by $58 \%$. The newly created category, Insufficiently known, includes $33 \%$ of the herpetofauna.

KEYwords: Conservation status, threats, amphibians, reptiles, Argentina.

\section{INTRODUCCION}

La legislación argentina, a través de la Ley 22421 (1981), determina que la fauna silvestre debe estar categorizada según su estado de conservación. La categorización oficial vigente (Secretaría de Agricultura y Ganadería 1983) fue realizada por dos especialistas de reconocido prestigio, pero no ha quedado registro de los criterios aplicados.

Siguiendo la tendencia mundial de utilizar criterios explícitos, objetivos y cuantificables, en 1993 la Subsecretaría de Recursos Naturales de la Nación decidió actualizar la categorización mencionada, para lo cual encomendó la tarea a la 
Dirección Nacional de Fauna y Flora Silvestres. Esta dirección determinó realizarla con el concurso de especialistas, adoptando el método de Reca et al. (1994), por medio del cual se arriba a un índice cuantitativo orientador que permite clasificar a las especies en las categorías de conservación que fija la ley. De esta manera hasta el momento se actualizó la clasificación de la fauna silvestre patagónica (Ubeda \& Grigera 1995) y de la mastofauna y herpetofauna argentinas (Reca et al. 1996, Lavilla et al. 2000).

En este trabajo se analizan los resultados de la categorización de la herpetofauna de Argentina últimamente realizada en el país (Lavilla et al. 2000) $\mathrm{y}$ de los factores determinantes del estatus asignado a las especies, con énfasis en las categorías que indican una mayor amenaza para la conservación de las especies. Además, se comparan los resultados de esta nueva evaluación con la clasificación oficial vigente de la herpetofauna de Argentina y con el estatus de conservación en los países limítrofes de las especies compartidas.

\section{MATERIALES Y METODOS}

a) ANÁlISIS DE LOS RESULTADOS DE LA RECATEGORIZACIÓN

En primer lugar se analizó la categorización de las especies y subespecies de manera general y por grupo taxonómico. Luego se analizaron particularmente aquellas especies y subespecies que fueron clasificadas En Peligro de Extinción, Amenazadas y Vulnerables. Para cada taxón se analizó la ponderación numérica de algunas variables que componen el índice utilizado, efectuada por los especialistas que evaluaron los distintos grupos de herpetozoos. Las variables consideradas son las siguientes:

-Distribución nacional (tamaño del área de distribución del taxón dentro del territorio argentino).

-Amplitud en el uso del hábitat (brinda una idea de la plasticidad de las especies en cuanto al hábitat). -Acciones extractivas (según el método utilizado pondera las acciones antrópicas que implican extracción de ejemplares de las poblaciones; los especialistas que evaluaron a los anfibios agregaron a esta variable la ponderación de la pérdida irreversible del hábitat). Se relacionó el tamaño corporal de las especies con las acciones extractivas que las afectan.

-Grado de protección a nivel nacional (dado por la protección efectiva en áreas protegidas).

Según el método utilizado (Reca et al. 1994), en la ponderación numérica de las variables el valor más alto representa la situación más adversa para la especie.

Por otra parte, se compilaron las amenazas de origen antrópico que afectan a los taxa evaluados, que son mencionadas en diversas fuentes bibliográficas (Freiberg 1970, 1979, Gallardo 1979, Centro Editor de América Latina 1983-1986, Gruss \& Waller 1988, Ortiz 1988, Chani et al. 1989, Yanosky 1989, Bertonatti \& González 1992, Bertonatti \& Banchs 1993, Bertonatti 1994, 2000, 2001, Chébez 1994, Serret \& Bertonatti 1994, Christie 1995a, 1995b, Avila \& Acosta 1996, Cabrera 1996, Di Tada et al. 1996, Reati 1996, Cabrera 1998, Ubeda et al. 1998, 1999, Lavilla 2000, 2001 Lavilla et al. 2000, Bertonatti \& Corcuera 2001). Las amenazas compiladas se tipificaron siguiendo la estructura jerárquica utilizada en la documentación de las listas rojas de la UICN del año 2000.

b) COMPARACIÓN DE LA NUEVA CATEGORIZACIÓN CON LA PREVIA

Se comparó el número de taxa evaluados y las categorías de conservación asignadas.

Las categorías utilizadas en 1983, determinadas por el Decreto 691 (1981) son: Amenazada de Extinción, Vulnerable, Rara, Indeterminada y No Amenazada. En la nueva categorización se utilizaron las categorías fijadas por el Decreto 666 (1997), modificatorio del anterior: En Peligro de Extinción, Amenazada, Vulnerable, No Amenazada e Insuficientemente Conocida.

Para poder efectuar la comparación se establecieron equivalencias entre las categorías utilizadas en cada caso, de acuerdo a sus definiciones (Tabla I). No existe una equivalencia estricta entre las categorías Rara, del Decreto 691 (1981) y Vulnerable, del Decreto 666 (1997), aunque su significado es similar.

Para conocer el estatus de conservación en Chile, Bolivia, Paraguay y Uruguay de la herpe- 
tofauna que se considera amenazada compartida con Argentina, se consultó a Glade (1993), Ergueta \& de Morales (1996), Núñez et al. (1997), Dirección de Parques Nacionales y Vida Silvestre (1997), Servicio Agrícola y Ganadero (1998), Morales \& Carreira (2000) y Maneyro \& Langone (2001).

TABla I. Categorías de conservación de uso oficial en Argentina. Equivalencias propuestas por las autoras entre las categorías fijadas por los Decretos 691 (1981) y 666 (1997). = la equivalencia es aproximada. *= en el Anexo I de la Resolución 144/83 (Secretaría de Agricultura y Ganadería 1983) esta categoría figura como En Peligro de Extinción.

TABLE I. Conservation categories used officially in Argentina. Equivalencies proposed by the authors between categories established by Decrees 691 (1981) and 666 (1997). = the equivalence is approximate. *= in Annex I, Resolution 144/ 83 (Secretaría de Agricultura y Ganadería, 1983) this category figures as In Danger of Extinction.

\begin{tabular}{ccc}
\hline Decreto $691 / 81$ & $=$ & Decreto 66697 \\
\hline Amenazada de Extinción & En Peligro de Extinción \\
Vulnerable & Amenazada \\
Rara & Vulnerable \\
No Amenazada & No Amenazada \\
Indeterminada & Insuficientemente conocida \\
\hline
\end{tabular}

\section{RESULTADOS}

A) ANÁLISIS DE LOS RESUlTAdOS DE LA RECATEGORIZACIÓN

De las 484 especies y subespecies de anfibios y reptiles calificadas en Lavilla et al. (2000), el $23.5 \%$ (114 taxa) se encuentra en categorías que implican riesgo para su conservación (En Peligro de Extinción, Amenazada o Vulnerable) (Apéndice, Tabla II).
Analizando separadamente las dos clases de herpetozoos, los anfibios se encuentran en mayor proporción en estas categorías que los reptiles (31.6\% y $19.2 \%$, respectivamente) (Tabla II). Un análisis más detallado por grupo taxonómico revela que los yacarés y las tortugas son los grupos que tienen mayor proporción de especies en situación de riesgo (100\% y $64.3 \%$ respectivamente), mientras que los saurios poseen la menor proporción $(8.4 \%)$.

TABLA II. Estado de conservación de la herpetofauna de Argentina. Datos tomados de Lavilla et al. (2000). Las cifras entre paréntesis indican porcentajes. EP= En Peligro de Extinción, A= Amenazada, V= Vulnerable, IC= Insuficientemente Conocida, NA= No Amenazada.

TABLE II. Conservation status of herpetofauna in Argentina. Data from Lavilla et al. (2000). The numbers between brackets indicate percentages. EP= In Danger of Extinction, A= Threatened, V=Vulnerable, IC= Insufficiently known, NA= Not threatened.

\begin{tabular}{lccccccc}
\hline \multicolumn{7}{c}{ Nümero de taxa (\%) } \\
\hline EP & A & V & $\begin{array}{c}\text { SUBTOTAL. } \\
\text { EP+A+V }\end{array}$ & IC & NA & $\begin{array}{c}\text { NDMEROS } \\
\text { TOTALES }\end{array}$ \\
\hline ANFIBIOS & $2(1.2)$ & $2(1.2)$ & $50(29.2)$ & $54(31.6)$ & $36(21.1)$ & $81(47.4)$ & 171 \\
REPTILES & $11(3.5)$ & $12(3.8)$ & $37(11.8)$ & $60(19.2)$ & $126(40.3)$ & $127(40.6)$ & 31.3 \\
Tortugas & 6 & 2 & 1 & 9 & 3 & 2 & 14 \\
Yacaris & 0 & 0 & 2 & 2 & 0 & 0 & 2 \\
Saurios & 2 & 5 & 7 & 14 & 108 & 45 & 167 \\
Serpientes & 3 & 5 & 27 & 35 & 15 & 80 & 130 \\
TOTALES & $13(2.7)$ & $14(2.9)$ & $87(18)$ & $114(23.6)$ & $162(33.5)$ & $208(43)$ & 484 \\
\hline
\end{tabular}


El $33 \%$ de los herpetozoos de Argentina (162 especies) fue asignado a la Categoría Insuficientemente Conocida (Tabla II). Esta categoría comprende el 40,2\% de los reptiles y el $21 \%$ de los anfibios.

La situación de los taxa calificados en las categorías que representan una mayor amenaza, en cuanto a las variables seleccionadas para ser analizadas en detalle, es la siguiente:
- Área de distribución en Argentina

El 88.9\% (48 taxa) de los anfibios con categorías más críticas y el $68.3 \%$ (41 taxa) de los reptiles en igual situación tienen áreas de distribución restringidas en Argentina, llegando a niveles de microendemismos (Fig. 1a). Ninguna especie cuya conservación se encuentra amenazada tiene amplia distribución en el país.

Entre los reptiles, el grupo que presenta distribuciones más restringidas es el de los ofidios, seguido por los saurios (Fig. 1b). a

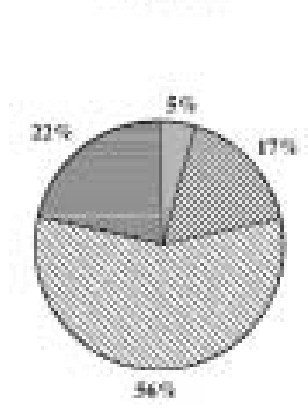

Herfeluzuns
Anfibios

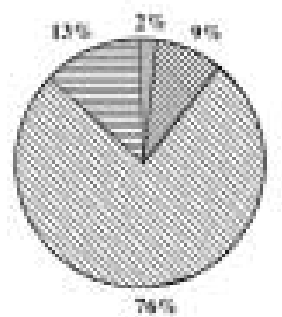

Repriles

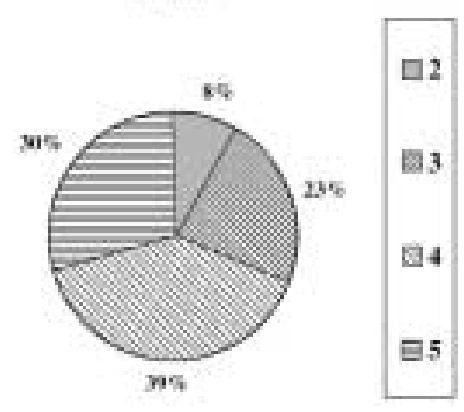

b

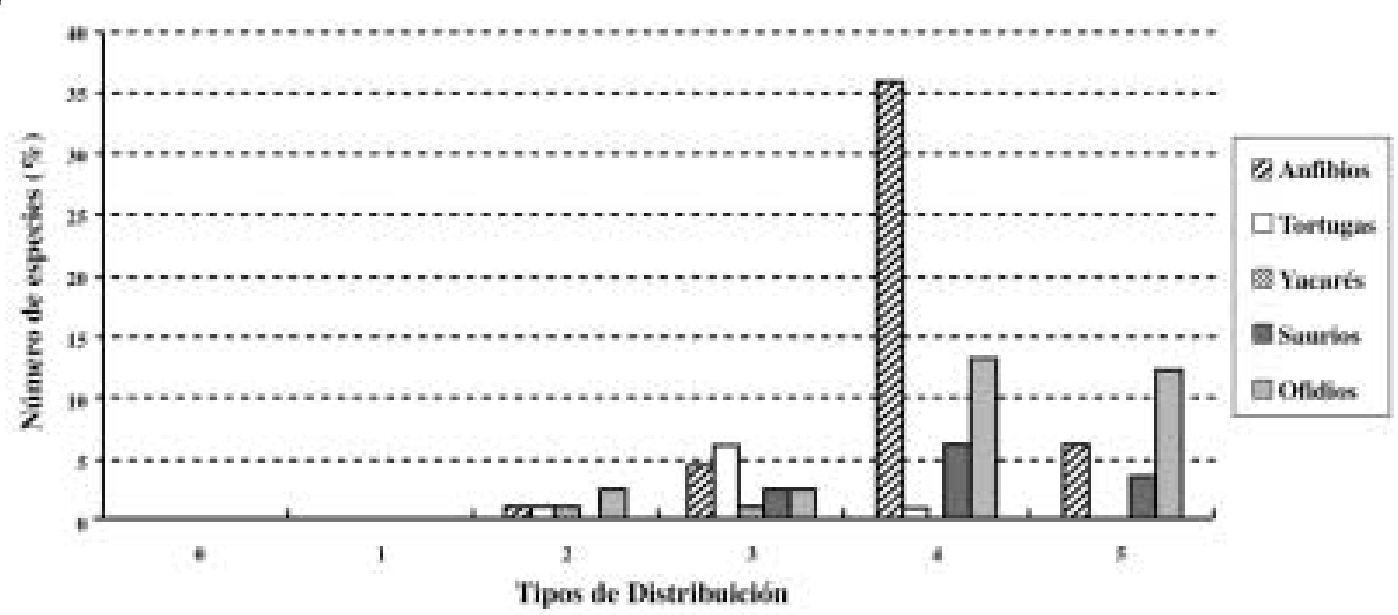

FIgURA 1. Distribución geográfica de los herpetozoos de Argentina cuya conservación se encuentra amenazada. Ponderación tomada de Lavilla et al. (2000). a)Herpetozoos, Anfibios y Reptiles, b)Anfibios y órdenes de Reptiles. $2=$ se distribuye en menos de la mitad del país, $3=$ distribución restringida, $4=$ muy localizada 0 endemismo, $5=$ microendemismo.

FIGURE 1. Geographical distribution of herpetofauna in Argentina whose conservation is threatened. Ponderations from Lavilla et al. (2000). a) Herpetofauna, Amphibians and Reptiles, b) Amphibians and orders of Reptiles. $2=$ distribution over less than half the country, $3=$ restricted distribution, $4=$ highly local distribution or endemism, $5=$ microendemism. 
- Amplitud en el uso de hábitat

De acuerdo a la ponderación de esta variable efectuada por los especialistas, los anfibios son el grupo que presenta mayores restricciones en el uso del hábitat (Fig. 2a). En las tortugas palustres el requerimiento de más de un ambiente a lo largo del ciclo de vida motivó una alta ponderación (Richard et al. en Lavilla et al. 2000) (Fig. 2b). a

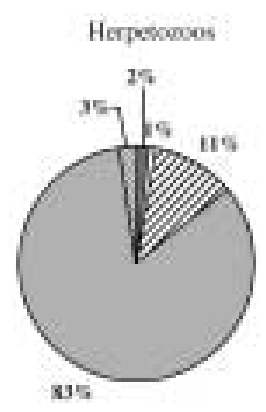

b

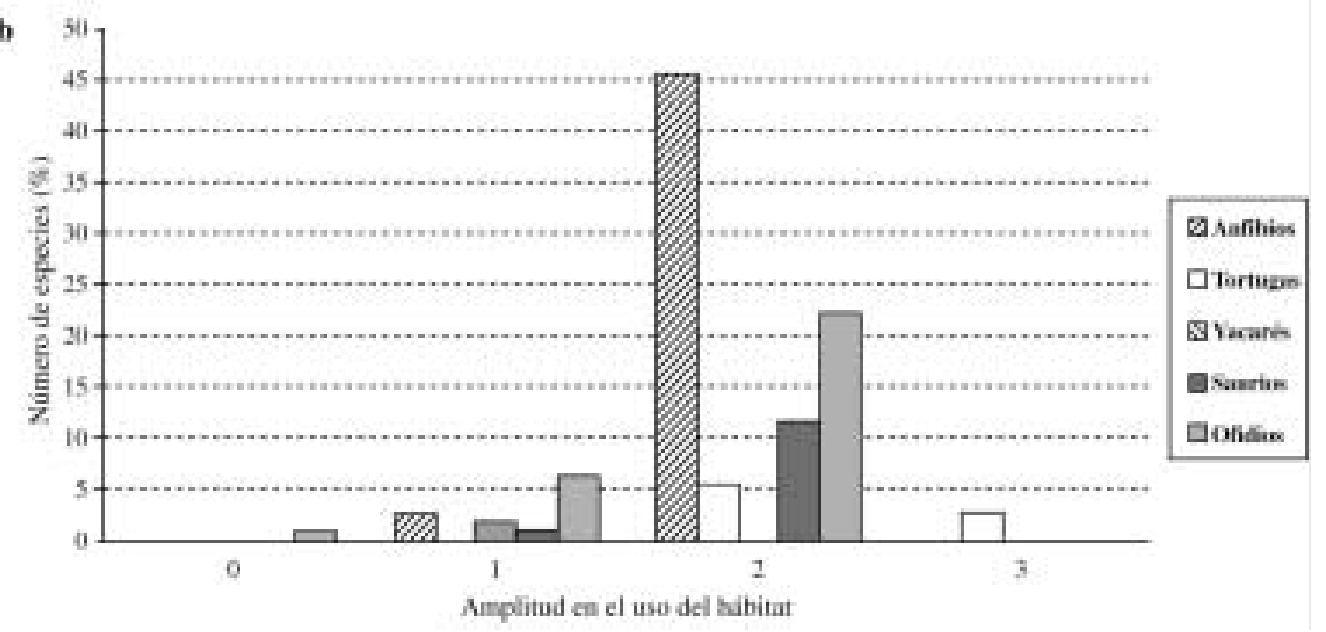

Figura 2. Amplitud en el uso del hábitat de los herpetozoos amenazados de Argentina. Ponderación tomada de Lavilla et al. (2000). a)Herpetozoos, Anfibios y Reptiles, b)Anfibios y órdenes de Reptiles. ?= se desconoce, $0=$ puede utilizar cuatro o más ambientes, $1=$ puede utilizar dos o tres ambientes, $2=$ puede utilizar sólo un ambiente o necesita más de uno, $3=$ necesita más de un ambiente.

FIGURE 2. Extent of use of habitat by threatened herpetofauna in Argentina. Ponderations from Lavilla et al. (2000). a) Herpetofauna, Amphibians and Reptiles, b) Amphibians and orders of Reptiles. ?= unknown, $0=$ can use four or more environments, $1=$ can use two or three environments, $2=$ can use only one environment or needs more than one, $3=$ needs more than one environment.

- Acciones extractivas

Sobre los 114 taxa listados en categorías de riesgo, 69 de ellos $(60.5 \%)$ son afectados en distinto grado por las acciones antrópicas comprendidas en la variable Acciones extractivas (Fig. 3a). Mientras los anfibios muestran niveles bajos o inexistentes de extracción, los reptiles presentan una mayor proporción de especies afectadas y una mayor intensidad de las acciones. Todas las tortugas y ofidios presentan problemas en distinto grado (Fig. 3b). 
a

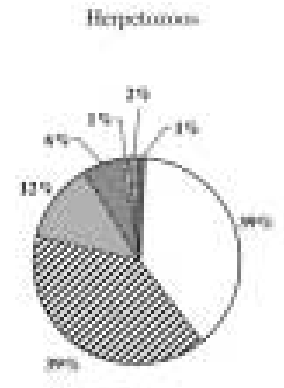

Herpotrowe
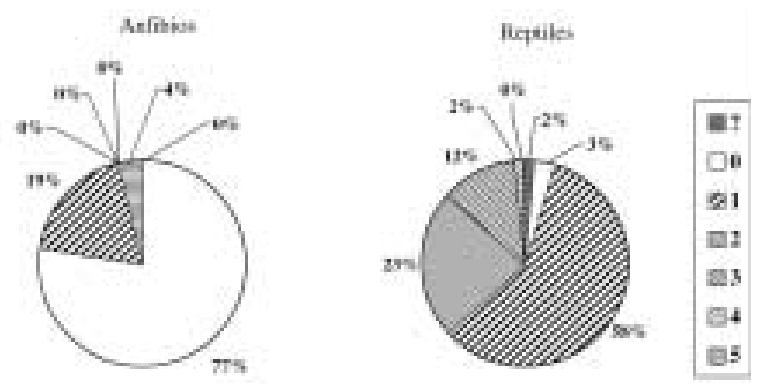

b

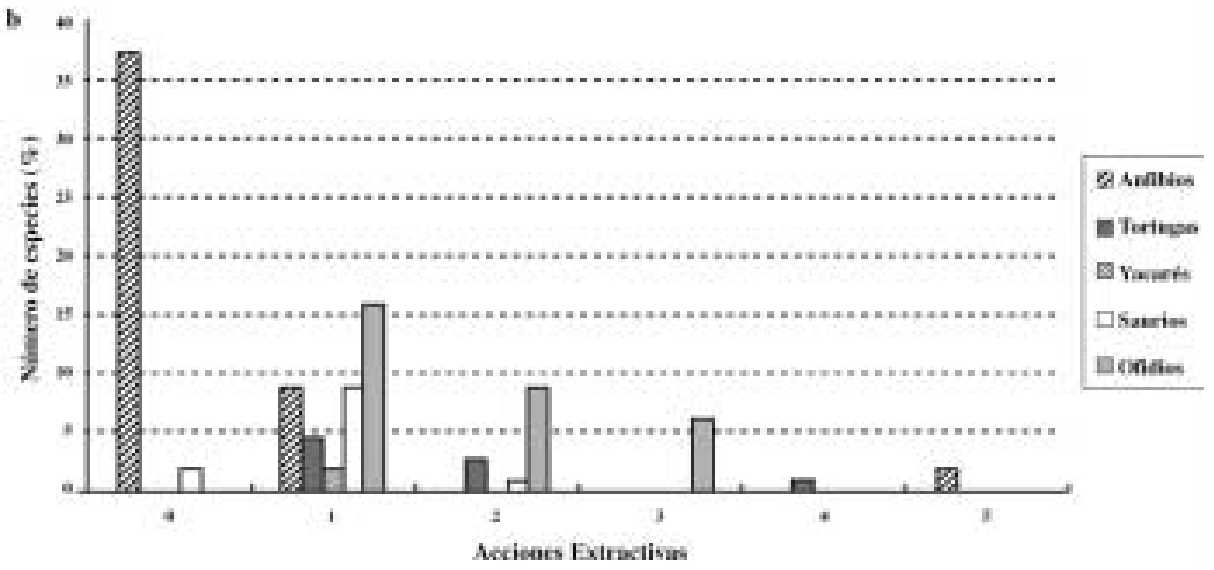

Figura 3. Acciones extractivas de los herpetozoos amenazados de Argentina. Ponderación tomada de Lavilla et al. (2000). a) Herpetozoos, Anfibios y Reptiles, b)Anfibios y órdenes de Reptiles. ?= se desconoce, 0= no existe extracción, 1= extracción por temor, repulsión, ser considerado perjudicial, aprovechamiento a pequeña escala o uso de subproductos, $2=$ caza deportiva o comercial a mediana escala, $3=$ explotación por dos o más de los motivos anteriores, 4= explotación intensiva, $5=$ alteración irreversible del hábitat.

FIGURE 3. Extractive actions of threatened herpetofauna in Argentina. Ponderations from Lavilla et al. (2000). a) Herpetofauna, Amphibians and Reptiles, b) Amphibians and orders of Reptiles. ?= unknown, $0=$ no extraction, $1=$ extraction due to fear, disgust, considering it harmful, small-scale consumption or use of sub-products, $2=$ medium-scale sport or commercial hunting, $3=$ exploited for two or more of the previous reasons, $4=$ intensive exploitation, $5=$ irreversible alteration of habitat.

- Grado de protección

El 78,2 \% de los anfibios (42 taxa) y el 78,3\% de los reptiles (47 taxa) en categorías de mayor amenaza no está protegido o está protegido por sólo una unidad de conservación (Fig. 4a). Dieciséis anfibios y 22 reptiles carecen de protección en Argentina. El grupo de las tortugas, especialmente las marinas, es el que posee menor grado de protección en el país (Fig. 4b). La mayoría de anfibios y ofidios tienen un grado de protección que podría calificarse de escaso a moderado, ya que están protegidos por 1 ó 2 unidades de conservación.

En la Tabla III se compilan los tipos de amenazas de origen antrópico, que han sido explícitamente citadas en la bibliografía, para los herpetozoos ubicados en categorías que indican situación de riesgo. De su análisis surge que de los 54 anfibios en esta situación, 18 de ellos $(33,3 \%)$ sufren algún tipo de amenaza directa o indirecta. De los 60 reptiles en igual situación, sobre 56 taxa $(93,3 \%)$ se han identificado algún tipo de amenaza.

La mayoría de los taxa analizados están afectados por acciones antrópicas que conducen a la alteración del hábitat. Asimismo, una proporción importante de especies está sometida a comercialización o a caza de subsistencia. Algunos autores señalan que la ganadería afecta por pisoteo o por competencia a algunos anfibios y tortugas terrestres, y otros que la introducción de especies acuáticas ha resultado en la declinación de algunos anfibios. 
Conservation of amphibians and reptiles from Argentina: UBEDA, C. \& D. GRIGERA

Tabla III. Factores de amenaza explícitamente citados para los herpetozoos cuya conservación se encuentra amenazada según Lavilla et al. (2000). Ef. In.: Efectos indirectos; Co: Contaminación; Tipificación de las amenazas según UICN. 1= hasta 1990, 2= actualmente la explotación es furtiva, 3= en el pasado reciente. AH: Alteración del Hábitat; AA: Actividad Agropecuaria; G: Ganadería (competencia trófica o pisoteo); FE: Forestación con especies exóticas; M: Minería; D: Deforestación; I: Industrias; CR: Construcción de Represas; U: Urbanización; FH: Fragmentación del Hábitat; F: Fuego; CS: Caza de subsistencia; PT: Persecución por temor, repugnancia o diversión; C: Comercialización; C: Captura accidental (en redes o de ejemplares varados); DM: Deportes de montaña / Tránsito vehículo todo terreno; E: Especies Exóticas; C: Contaminación.

Table III. Explicitly cited threats to herpetofauna whose conservation is threatened, according to Lavilla et al. (2000). Ef. In.: Indirect effects; Co: Contamination; Typification of threats according to IUCN. 1= up to 1990, 2= currently poached, $3=$ in the recent past. AH: Habitat alteration; AA: Agropecuarian Activity; G: Stock Farm (trophic competence or trample); FE: Forestation with exotic species; M: Mining; D: Deforestation; I: Industries; CR: Dams construction; U: Urbanization; FH: Habitat fragmentation; F: Fire; CS: Subsistency hunting; PT: Persecution; C: Commercialization; C: Accidental Capture (in nests or other); DM: Mountain sports; E: Exotic species; C: Pollution.

\begin{tabular}{|c|c|c|c|c|c|c|c|c|c|c|c|c|c|c|c|c|c|}
\hline \multirow[b]{2}{*}{ ESPECIE/SUBESPECIE } & \multicolumn{10}{|c|}{ Pérdida del hábitat primariamente antrópica } & \multicolumn{4}{|c|}{$\begin{array}{c}\text { Pérdida directa / } \\
\text { Explotación }\end{array}$} & \multicolumn{2}{|c|}{ Ef. In. } & \multirow{2}{*}{$\frac{\mathrm{Co}}{\mathrm{C}}$} \\
\hline & $\mathrm{AH} \mathrm{AA}$ & G & $\mathrm{EE}$ & M & $\mathrm{D}$ & I & $\mathrm{CR}$ & $\mathrm{U}$ & $\mathrm{FH}$ & $\mathrm{F}$ & CS & PT & $\mathrm{C}$ & C & $\mathrm{DM}$ & $\mathrm{E}$ & \\
\hline \multicolumn{18}{|l|}{ ANFIBIOS } \\
\hline Alsodes gargolaneuquensis & & & & & & & & & & & & & & & & $\mathrm{x}$ & \\
\hline Atelognathus nitoi & & & & & & & & & & $\mathrm{x}$ & & & & & $\mathrm{x}$ & & \\
\hline Atelognathus patagonicus & & & & & & & & & & & & & & & & $\mathrm{x}$ & \\
\hline Atelognathus salai & & $\mathrm{x}$ & & & $\mathrm{x}$ & & & & & & & & & & & & \\
\hline Bufo achalensis & & $\mathrm{x}$ & & & & & & & $\mathrm{x}$ & & & & & & & & \\
\hline Bufo arenarummendocinus & & & & & & & & & & & & $\mathrm{x}$ & & & & & \\
\hline Gastrotheca christiani & $\mathrm{x}$ & & & $\mathrm{x}$ & & & & & & & & & & & & & \\
\hline Hyalinobatrachium uranoscopum & & & & & & & & & $\mathrm{x}$ & & & & & & & & \\
\hline Leptodactylus laticeps & & & & & & & & & & & & & $\mathrm{x}$ & & & & \\
\hline Melanophryniscus stelzneri stelzneri & & & $\mathrm{x}$ & & & & & & & & & & & $\mathrm{x}$ & & & \\
\hline Odontophrynus achalensis & & & & & & & & & $\mathrm{x}$ & & & & & & & & \\
\hline Pleurodema kriegi & & & & & & & & & $\mathrm{x}$ & & & & & & & & \\
\hline Somuncuria somuncurensis & & $\mathrm{x}$ & & & & & & & & & & & & & & $\mathrm{x}$ & $\mathrm{x}$ \\
\hline Telmatobius atacamensis & $\mathrm{x}$ & $\mathrm{x}$ & & & & & & & & & $\mathrm{x}$ & & & & $\mathrm{x}$ & $\mathrm{x}$ & $\mathrm{x}$ \\
\hline Telmatobius hauthali & $\mathrm{x}$ & $\mathrm{x}$ & & $\mathrm{x}$ & & & & & & & & & & & $\mathrm{x}$ & & $\mathrm{x}$ \\
\hline Telmatobius laticeps & $\mathrm{x}$ & $\mathrm{x}$ & & $\mathrm{x}$ & & & & & & & & & & & $\mathrm{x}$ & & $\mathrm{x}$ \\
\hline Telmatobius pisanoi & $\mathrm{x}$ & $\mathrm{x}$ & & $\mathrm{x}$ & & & & & & & & & & & $\mathrm{x}$ & & $\mathrm{x}$ \\
\hline \multicolumn{18}{|l|}{ Telmatobius schreiteri } \\
\hline \multicolumn{18}{|l|}{ TORTUGAS } \\
\hline Acanthochelys pallidipectoris & $\mathrm{x}$ & & & & $\mathrm{x}$ & & & & & & & & $\mathrm{x}$ & & & & \\
\hline Acanthochelys spixi & $\mathrm{x}$ & & & & & & & & & & & & & & & & $\mathrm{x}$ \\
\hline Carettacaretta & & & & & & & & & & & & & & $\mathrm{x}$ & & & \\
\hline Cheloniamydas & & & & & & & & & & & & & & $\mathrm{x}$ & & & \\
\hline Chelonoidis carbonaria & $\mathrm{x}$ & $\mathrm{x}$ & & & $\mathrm{x}$ & $\mathrm{x}$ & & & & $\mathrm{x}$ & $\mathrm{x}$ & & $\mathrm{x}$ & & & & \\
\hline Chelonoidis chilensis & & $\mathrm{x}$ & & & $\mathrm{x}$ & & & & & & $\mathrm{x}$ & & $\mathrm{x}$ & & & & \\
\hline Chelonoidis donosobarrosi & $\mathrm{x}$ & $\mathrm{x}$ & & & & $\mathrm{x}$ & & & & $\mathrm{x}$ & $\mathrm{x}$ & & $\mathrm{x}$ & & & & \\
\hline Dermochelys coriacea & & & & & & & & & & & & & & $\mathrm{x}$ & & & \\
\hline Trachemys scripta dorbignyi & $\mathrm{x}$ & & & & & & & & & & & & $\mathrm{x}$ & & & & \\
\hline \multicolumn{18}{|l|}{ COCODRILOS } \\
\hline Caiman latirostris & & & & & & & $\mathrm{x}$ & & & & $\mathrm{x}$ & & $\mathrm{x}(1)$ & & & & \\
\hline Caiman yacare & & & & & & & $\mathrm{x}$ & & & & $\mathrm{x}$ & & $\mathrm{x}(1)$ & & & & \\
\hline \multicolumn{18}{|l|}{ SAURIOS } \\
\hline Cnemidophorus lacertoides & $\mathrm{x}$ & & & & & & & $\mathrm{x}$ & & & & & $\mathrm{x}$ & & & & \\
\hline Cnemidophorus serranus & $\mathrm{x}$ & & & & & & & $\mathrm{x}$ & & & & & $\mathrm{x}$ & & & & \\
\hline
\end{tabular}


Gayana 67(1), 2003

Continuación Tabla III.

\begin{tabular}{|c|c|c|c|c|c|c|c|c|c|c|c|c|c|c|c|c|c|}
\hline \multirow[b]{2}{*}{ ESPECIE/SUBESPECIE } & \multicolumn{10}{|c|}{ Pérdida del hábitat primariamente antrópica } & \multicolumn{4}{|c|}{$\begin{array}{l}\text { Pérdida directa / } \\
\text { Explotación } \\
\end{array}$} & \multicolumn{2}{|c|}{ Ef. In. } & \multirow{2}{*}{$\frac{\mathrm{Co}}{\mathrm{C}}$} \\
\hline & $\mathrm{AH} \mathrm{AA}$ & G & $\mathrm{FE}$ & M & $\mathrm{D}$ & I & $\mathrm{CR}$ & $\mathrm{U}$ & $\mathrm{FH}$ & $\mathrm{F}$ & CS & $\mathrm{PT}$ & C & $\mathrm{C}$ & $\mathrm{DM}$ & $\mathrm{E}$ & \\
\hline Diplolaemus leopardinus & & & & & & & & & & & & & $?$ & & & & \\
\hline Leiosaurus paronae & $\mathrm{x}$ & & & & $\mathrm{x}$ & & $\mathrm{x}$ & & & & & & & & & & \\
\hline Liolaemusmultimaculatus & & & $\mathrm{x}$ & & & & & $\mathrm{x}$ & & & & & $\mathrm{x}$ & & & & \\
\hline Liolaemus rabinoi & $\mathrm{x}$ & & & & & & & & & & & & & & & & \\
\hline Polychrus acutirostris & & & & & $\mathrm{x}$ & & $\mathrm{x}$ & & & & & & & & & & \\
\hline Pristidactylus achalensis & & $\mathrm{x}$ & & & & & & & & & & & $\mathrm{x}$ & & $\mathrm{x}$ & & \\
\hline Pristidactylus casuhatiensis & $\mathrm{x}$ & & & & & & & & & & & & & & & & \\
\hline Urostrophus gallardoi & & & & & $\mathrm{x}$ & & $\mathrm{x}$ & & & $\mathrm{x}$ & & & $?$ & & & & \\
\hline \multicolumn{18}{|l|}{ OFIDIOS } \\
\hline Atractus paraguayensis & & & & & & & & & & & & $\mathrm{x}$ & & & & & \\
\hline Atractus reticulatus & & & & & & & & & & & & $\mathrm{x}$ & & & & & \\
\hline Boaconstrictoroccidentalis & $\mathrm{x}$ & & & & & & & & & & $\mathrm{x}$ & $\mathrm{x}$ & $x(2)$ & & & & \\
\hline Bothrops cotiara & & & & & & & & & $\mathrm{x}$ & & & $\mathrm{x}$ & & & & & \\
\hline Bothropsjararacussu & $\mathrm{x}$ & & & & & & & & & & & $\mathrm{x}$ & & & & & \\
\hline Bothrops moojeni & & & & & & & & & & & & $\mathrm{x}$ & & & & & \\
\hline Chironius bicarinatus & & & & & & & & & & & & $\mathrm{x}$ & & & & & \\
\hline Chironius exoletus & & & & & & & & & & & & $\mathrm{x}$ & & & & & \\
\hline Cleliaplumbea & & & & & & & & & & & & $\mathrm{x}$ & & & & & \\
\hline Dipsas indicabucephala & & & & & & & & & & & & $\mathrm{x}$ & & & & & \\
\hline Dipsas indica cisticeps & & & & & & & & & & & & $\mathrm{x}$ & & & & & \\
\hline Echinanthera cyanopleura & & & & & $\mathrm{x}$ & & & & & & & $\mathrm{x}$ & & & & & \\
\hline Epicrates cenchriaalvarezi & & & & & & & & & & & & $\mathrm{x}(3)$ & & & & & \\
\hline Epicrates cenchria crassus & & & & & & & & & $\mathrm{x}$ & & & $\mathrm{x}$ & & & & & \\
\hline Erythrolamprus aesculapii venustissimu & & & & & & & & & & & & $\mathrm{x}$ & & & & & \\
\hline Eunectesnotaeus & & & & & & & $\mathrm{x}$ & & & & $\mathrm{x}$ & $\mathrm{x}$ & $\mathrm{x}$ & & & & \\
\hline Hydrops triangularis & & & & & & & & & & & & $\mathrm{x}$ & & & & & \\
\hline Imantodes cenchoa & & & & & $\mathrm{x}$ & & & & & & & $\mathrm{x}$ & & & & & \\
\hline Leptotyphlops albipunctus & & & & & & & & & & & & $\mathrm{x}$ & & & & & \\
\hline Leptotyphlops vellardi & & & & & & & & & & & & $\mathrm{x}$ & & & & & \\
\hline Liophis elegantissimus & & & & & & & & & & & & $\mathrm{x}$ & & & & & \\
\hline Liophis frenatus & & & & & & & & & & & & $\mathrm{x}$ & & & & & \\
\hline Liophis reginae macrosomus & & & & & & & & & & & & $\mathrm{x}$ & & & & & \\
\hline Liophis vanzolinii & & & & & & & & & & & & $\mathrm{x}$ & & & & & \\
\hline Micrurus lemniscatus & & & & & & & & & & & $\mathrm{x}$ & $\mathrm{x}$ & & & & & \\
\hline Oxyrhopus clathratus & & & & & & & & & & & & $\mathrm{x}$ & & & & & \\
\hline Oxyrhopus petola & & & & & & & & & & & & $\mathrm{x}$ & & & & & \\
\hline Phalotris reticulatus & & & & & & & & & & & & $\mathrm{x}$ & & & & & \\
\hline Pseudoboahaasi & & & & & & & & & $\mathrm{x}$ & & & $\mathrm{x}$ & & & & & \\
\hline Pseudoeryx plicatilis plicatilis & & & & & & & & & & & & $\mathrm{x}$ & & & & & \\
\hline Rachidelus brazili & & & & & & & & & & & & $\mathrm{x}$ & & & & & \\
\hline Sibynomorphus mikani & & & & & & & & & & & & $\mathrm{x}$ & & & & & \\
\hline Spilotes pullatus anomalepis & & & & & & & $\mathrm{x}$ & & & & & $\mathrm{x}$ & & & & & \\
\hline Tomodon dorsatus & & & & & & & & & & & & $\mathrm{x}$ & & & & & \\
\hline Xenodonneuwiedii & & & & & & & & & & & & $\mathrm{x}$ & & & & & \\
\hline
\end{tabular}


a

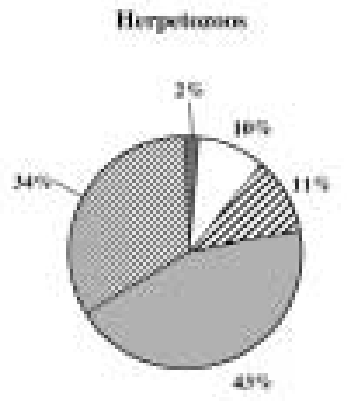

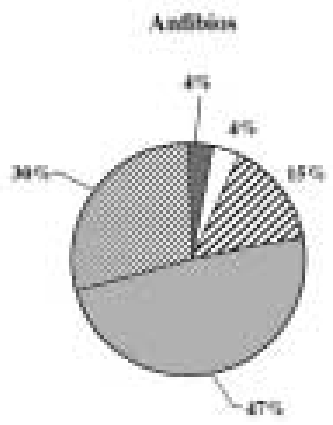

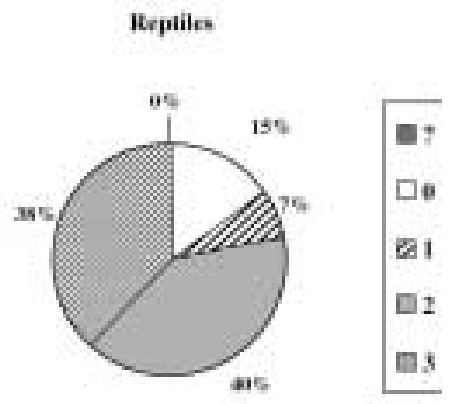

b.

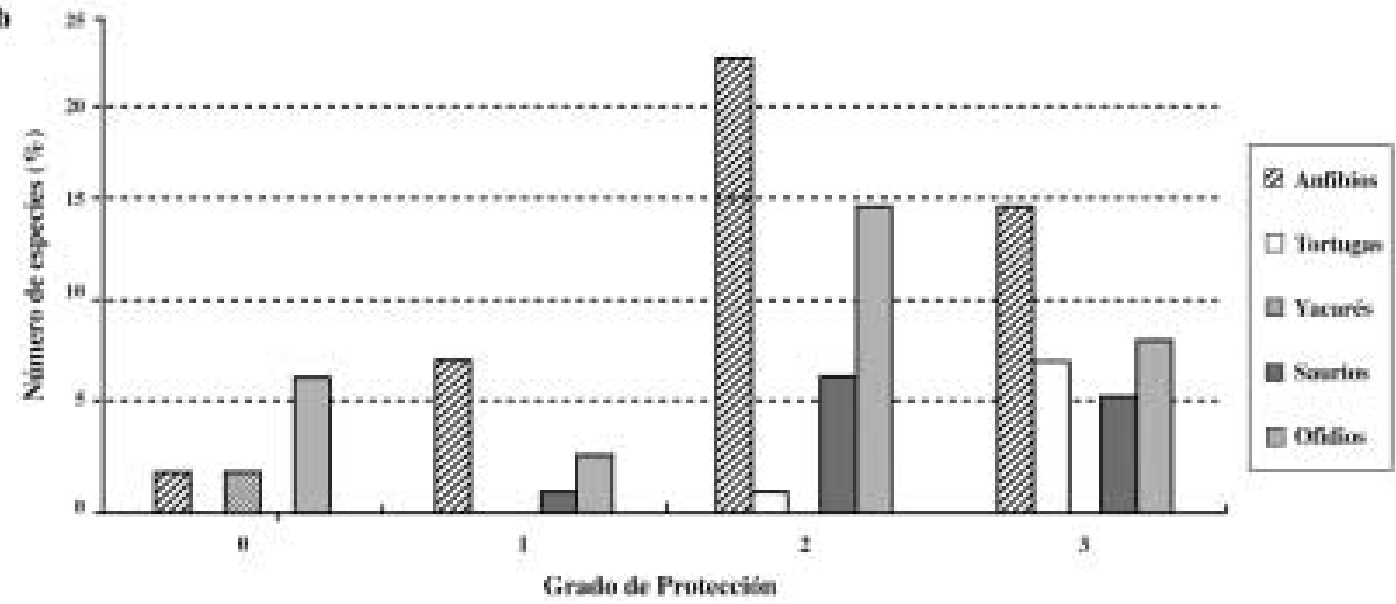

Figura 4. Grado de protección de los herpetozoos amenazados de Argentina. Ponderación tomada de Lavilla et al. (2000). a) Herpetozoos, Anfibios y Reptiles, b)Anfibios y órdenes de Reptiles. ?= se desconoce, $0=$ protegida por tres o más unidades de conservación, $1=$ protegida por dos unidades, $2=$ protegida por una unidad, $3=$ no protegida.

Figure 4. Degree of protection of threatened herpetofauna in Argentina. Ponderations from Lavilla et al. (2000). a) Herpetofauna, Amphibians and Reptiles, b) Amphibians and orders of Reptiles. ?= unknown, $0=$ protected by three or more conservation units, $1=$ protected by two units, $2=$ protected by one unit, $3=$ not protected.

\section{b) COMPARACIÓN DE LA NUEVA CATEGORIZACIÓN CON LA PREVIA}

La primera diferencia que se advierte al comparar la categorización del año 2000 (Tabla II) con la de 1983 (Tabla IV), es el número de taxa categorizados. En 1983 se evaluaron 318 herpetozoos (120 anfibios y 198 reptiles). Con el conocimiento actualizado de la herpetofauna argentina, en la recategorización se evaluaron 484 herpetozoos (171 anfibios y 313 reptiles), representando un incremento de taxa del 52\% (Tabla IV). Salvo en tortugas y yacarés, que han conservado el número de especies, hubo un aumento considerable en todos los grupos. Se incorporaron más taxa, en orden decreciente, en saurios, serpientes y anfibios.
La Figura 5 muestra la proporción de especies de cada categoría en ambos listados. En la categorización de 1983 hay una mayor proporción de especies en categorías de amenaza (En Peligro, Vulnerable, Rara o Indeterminada), mientras que en la actualizada aparece la categoría Insuficientemente Conocida con una proporción importante de especies, y hay una menor proporción de taxa No Amenazados.

En la Tabla V se indican las categorías de conservación asignadas a las especies que Argentina comparte con Chile, Bolivia y/o Uruguay, cuya conservación está amenazada en alguno de estos países. En esta situación se encuentran sólo dos espe- 
cies de Paraguay, Caiman latirostris y Bothrops jararacussu, por lo tanto no se incluyeron en la Tabla.
II

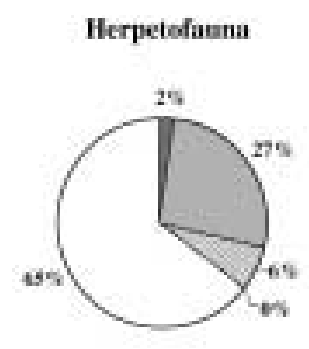

b

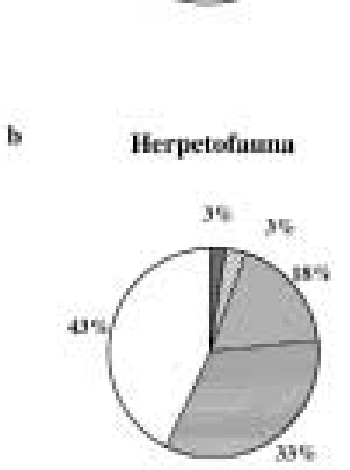

\section{.}

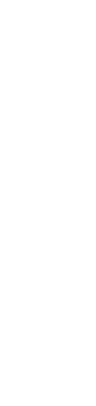

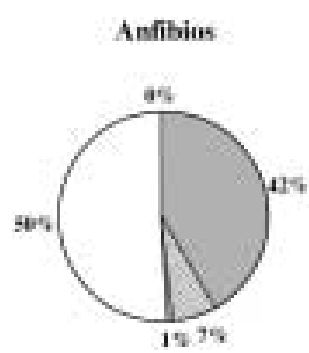

Anfibies

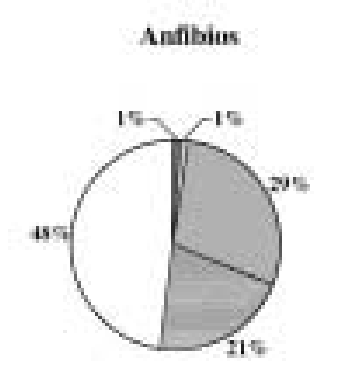

Aproximadamente la mitad $(45 \%)$ de las 89 especies o subespecies listadas, no están en riesgo en Argentina.

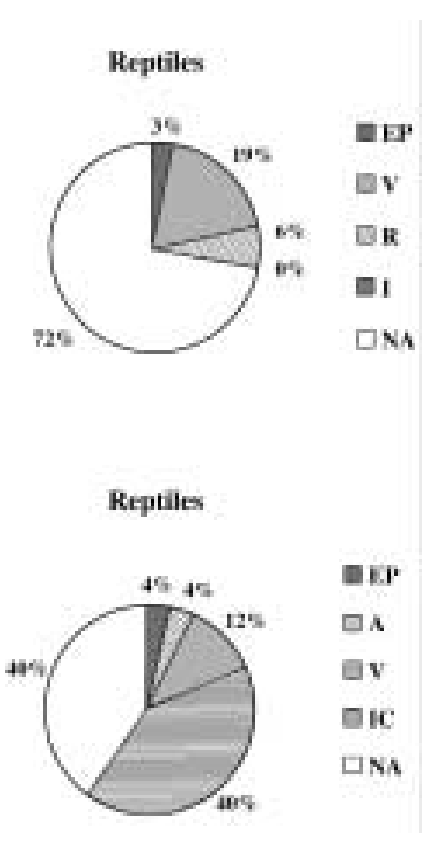

Figura 5. Proporción de taxa en las categorías de conservación según: a) Res. 144/83, Anexo I (Secretaría de Agricultura y Ganadería, 1983). EP= En Peligro, V= Vulnerable, $\mathrm{R}=\mathrm{Rara}$, I= Indeterminada, NA= No Amenazada. b) Lavilla et al. (2000). EP= En Peligro de Extinción, V= Vulnerable, A= Amenazada, IC= Insuficientemente Conocida, NA= No Amenazada. Ver equivalencias entre las categorías en la Tabla I.

FIGURE 5. Proportion of taxa in the conservation categories according to: a) Res. 144/83, Annex I (Secretaría de Agricultura y Ganadería, 1983). EP= Endangered, V= Vulnerable, $R=$ Rare, I= Undetermined, NA= Not Threatened.

b) Lavilla et al. (2000). EP= In Danger of Extinction, V=Vulnerable, A= Threatened, IC= Insufficiently Known, NA= Not Threatened. See equivalencies between categories in Table I.

TABLA IV. Comparación de la categorización vigente (Secretaría de Agricultura y Ganadería 1983, Resolución 144) y la categorización de Lavilla et al. (2000). Se indica en términos absolutos y porcentuales la diferencia en el número de taxa entre ambas categorizaciones.

TABle IV. Comparison of current categorization (Secretaría de Agricultura y Ganadería, 1983, Resolution 144) and categorization according to Lavilla et al. (2000). The difference in number of taxa between both categorizations is provided both in absolute numbers and percentages.

\begin{tabular}{lcccc}
\hline \multicolumn{5}{c}{ Nùmero de taxa } \\
\hline Res. 144/83 & $\begin{array}{c}\text { Lavilla et od. } \\
(2000)\end{array}$ & Diferencia & \% incremento \\
\hline Anfibios & 120 & 171 & 51 & 42.5 \\
Tortugas & 14 & 14 & 0 & 0 \\
Yacares & 2 & 2 & 0 & 0 \\
Saurios & 97 & 167 & 70 & 72 \\
Serpientes & 85 & 130 & 45 & 53 \\
TOTALES & 318 & 484 & 166 & 52 \\
\hline
\end{tabular}


Tabla V. Especies de la herpetofauna de Argentina compartidas con Chile, Bolivia, y/o Uruguay, cuya conservación se encuentra amenazada en alguno de estos países. Se indican las categorías que les fueron asignadas en cada país. Núñez et al. (1997) consignan las categorías por regiones y el Servicio Agrícola y Ganadero (1998) por Zonas de Caza, de modo que se indican todas las categorías asignadas al taxón. A= Amenazada, AE $=$ Atención Especial, $\mathrm{CR}=$ En Peligro Crítico, $\mathrm{DD}=$ Datos Insuficientes, $\mathrm{EP}=\mathrm{P}=$ En Peligro/de Extinción, F= Fuera de Peligro, $\mathrm{I}=\mathrm{IC}=$ Insificientemente/Inadecuadamente Conocida, $\mathrm{LR}=$ Menor Riesgo, MP= Máxima prioridad de conservación, NA= No Amenazada, $\mathrm{P}=$ Prioridad de Conservación, $\mathrm{R}=\mathrm{Rara}, \mathrm{V}=$ Vulnerable, $\mathrm{VU}=$ Vulnerable.

TABle V. Herpetofauna species in Argentina shared with Chile, Bolivia, and/or Uruguay, whose conservation is threatened in any of these countries. The categories assigned in each country are shown. Núñez et al. (1997) assign categories according to Regions and the Servicio Agrícola y Ganadero (1998) according to Hunting Zones; all the categories assigned to a taxon are shown. $\mathrm{A}=$ Threatened, $\mathrm{AE}=$ Special Attention, $\mathrm{CR}=\mathrm{Critically}$ Endangered, $\mathrm{DD}=$ Data Deficient, $\mathrm{EP}=\mathrm{P}=$ In danger of Extinction, $\mathrm{F}=$ Out of Danger, I= IC= Insufficiently / Inadequately Known, LR= Lower Risk, $\mathrm{MP}=$ Maximum Priority for conservation, $\mathrm{NA}=$ Not Threatened, $\mathrm{P}=$ Conservation Priority, $\mathrm{R}=\mathrm{Rare}, \mathrm{V}=$ $\mathrm{VU}=$ Vulnerable.

\begin{tabular}{|c|c|c|c|c|c|c|}
\hline & ARGENTINA & & CHILE & & BOLIVIA & URUGUAY \\
\hline $\begin{array}{l}\text { ESPECIE / } \\
\text { SUBESPECIE }\end{array}$ & $\begin{array}{l}\text { Lavilla et al. } \\
\quad(2000)\end{array}$ & $\begin{array}{l}\text { Glade } \\
\text { (1993) }\end{array}$ & $\begin{array}{c}\text { Nuñez et } \\
a l . \\
(1997)\end{array}$ & $\begin{array}{l}\text { SAG } \\
(1998)\end{array}$ & $\begin{array}{c}\text { Ergueta \& } \\
\text { de Morales } \\
\text { (1996) }\end{array}$ & $\begin{array}{c}\text { Maneyro \& } \\
\text { Langone } \\
(2001)\end{array}$ \\
\hline
\end{tabular}

ANFIBIOS

Alsodes monticola

(incluye coppingeri)

Alsodes verrucosus

Argenteohyla siemersi siemersi

Atelognathus aff. grandisonae

Batrachyla taeniata

Bufo rubropunctatus

Bufo spinulosus papillosus

Bufo spinulosus spinulosus

Bufo variegatus

Ceratophrys ornata

Chthonerpeton indistinctum

Eupsophus roseus

Eupsophus vertebralis

Hyla minuta

Hyla nana

Hylorina sylvatica

Leptodactylus chaquensis

Lysapsus limellius

Melanophryniscus devincenzii

Physalaemus fernandezae

Pleurodema bufoninum

Pleurodema marmoratum

Pleurodema thaul

Rhinoderma darwinii

Scinax berthae

Scinax fuscovaria

Scinax nasicus

Telmatobius marmoratus

$\mathrm{NA}$
$\mathrm{IC}$
$\mathrm{I}$
$\mathrm{IC}$
$\mathrm{N}$

REPTILES

TORTUGAS

Acanthochelys pallidipectoris

Caretta caretta

Chelonia mydas

Chelonoidis carbonaria

Chelonoidis chilensis

Dermochelys coriacea

YACARÉS

Caiman latirostris

Caiman yacare

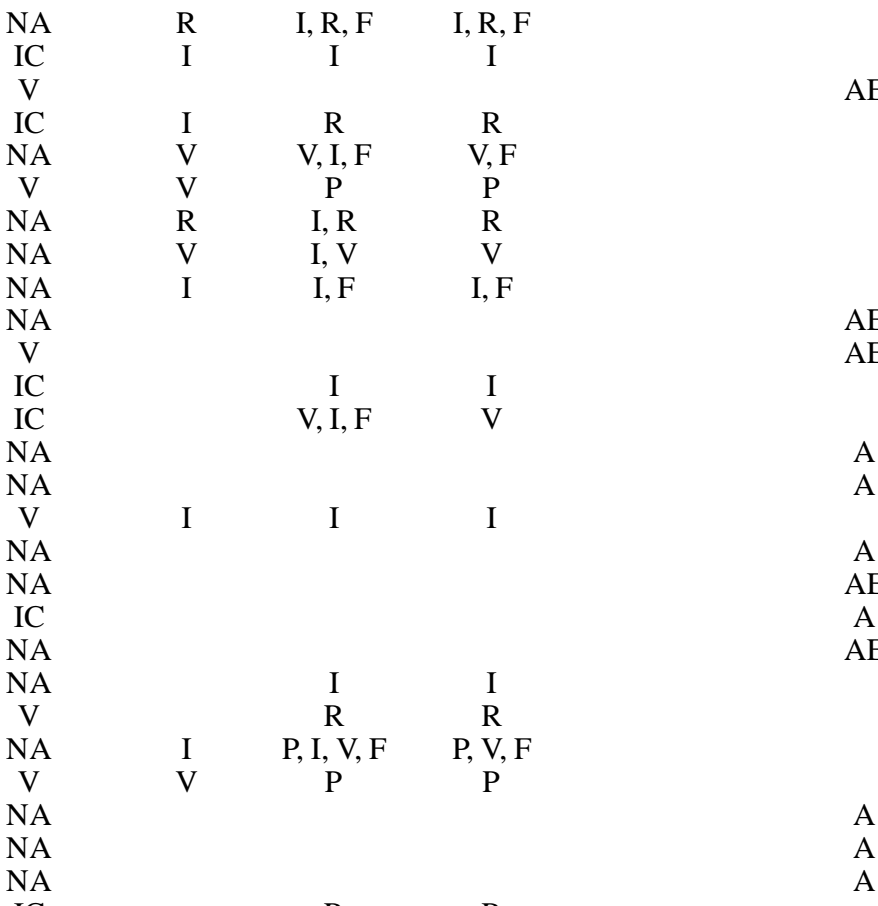

$\mathrm{R}$

$\mathrm{R}$
$\mathrm{AE}$

AE

A

A

A

$\mathrm{AE}$

AE

A

A

DD

VU

DD

CR

LR 
Gayana 67(1), 2003

Continuación Tabla V.

\begin{tabular}{|c|c|c|c|c|c|c|}
\hline & ARGENTINA & & CHILE & & BOLIVIA & URUGUAY \\
\hline $\begin{array}{l}\text { ESPECIE / } \\
\text { SUBESPECIE }\end{array}$ & $\begin{array}{l}\text { Lavilla et al. } \\
\quad(2000)\end{array}$ & $\begin{array}{l}\text { Glade } \\
\text { (1993) }\end{array}$ & $\begin{array}{c}\text { Nuñez et } \\
a l . \\
(1997)\end{array}$ & $\begin{array}{l}\text { SAG } \\
(1998)\end{array}$ & $\begin{array}{c}\text { Ergueta \& } \\
\text { de Morales } \\
(1996)\end{array}$ & $\begin{array}{c}\text { Maneyro \& } \\
\text { Langone } \\
(2001)\end{array}$ \\
\hline \multicolumn{7}{|l|}{ SAURIOS } \\
\hline Diplolaemus bibronii & IC & & $\mathrm{R}$ & $\mathrm{R}$ & & \\
\hline Diplolaemus darwinii & IC & & $\mathrm{R}$ & $\mathrm{R}$ & & \\
\hline Diplolaemus leopardinus & $\mathrm{V}$ & & I & I & & \\
\hline Liolaemus archeforus & IC & & $\mathrm{R}$ & $\mathrm{R}$ & & \\
\hline Liolaemus bibronii & NA & & $\mathrm{R}$ & $\mathrm{R}$ & & \\
\hline Liolaemus boulengeri & NA & & $\mathrm{R}$ & $\mathrm{R}$ & & \\
\hline Liolaemus buergeri & IC & & $\mathrm{R}$ & $\mathrm{R}$ & & \\
\hline Liolaemus ceii & IC & & $\mathrm{R}$ & $\mathrm{R}$ & & \\
\hline Liolaemus chiliensis & IC & $\mathrm{V}$ & I & I & & \\
\hline Liolaemus constanzae & IC & $\mathrm{R}$ & $\mathrm{R}$ & $\mathrm{R}$ & & \\
\hline Liolaemus dorbignyi & IC & I & $\mathrm{R}$ & $\mathrm{R}$ & & \\
\hline Liolaemus eleodori & IC & & $\mathrm{R}$ & $\mathrm{R}$ & & \\
\hline Liolaemus fitzgeraldi & IC & $\mathrm{R}$ & $\mathrm{R}$ & $\mathrm{R}$ & & \\
\hline Liolaemus fitzingerii & NA & I & $\mathrm{R}$ & $\mathrm{R}$ & & \\
\hline Liolaemus gravenhorstii & IC & $\mathrm{P}$ & $\mathrm{P}$ & $\mathrm{P}$ & & \\
\hline Liolaemus kingii & IC & $\mathrm{R}$ & $\mathrm{R}$ & $\mathrm{R}$ & & \\
\hline Liolaemus kriegi & IC & I & $\mathrm{I}, \mathrm{R}$ & $\mathrm{R}$ & & \\
\hline Liolaemus lemniscatus & IC & V & $\mathrm{V}, \mathrm{F}$ & $\mathrm{V}, \mathrm{F}$ & & \\
\hline Liolaemus lineomaculatus & IC & $\mathrm{R}$ & $\mathrm{R}$ & $\mathrm{R}$ & & \\
\hline Liolaemus magellanicus & IC & $\mathrm{R}$ & V & V & & \\
\hline Liolaemus monticola & IC & & $\mathrm{V}, \mathrm{R}, \mathrm{F}$ & $\mathrm{V}, \mathrm{R}$ & & \\
\hline Liolaemus nigriceps & IC & & V & V & & \\
\hline Liolaemus ornatus & IC & & $\mathrm{R}$ & $\mathrm{R}$ & & \\
\hline Liolaemus pictus pictus & IC & & $\mathrm{V}, \mathrm{F}$ & V & & \\
\hline Liolaemus rothi & IC & & V & V & & \\
\hline Liolaemus tenuis & IC & & $\mathrm{V}, \mathrm{F}$ & V & & \\
\hline Liolaemus vallecurensis & IC & & $\mathrm{R}$ & $\mathrm{R}$ & & \\
\hline Phymaturus flagellifer & IC & & $\mathrm{P}$ & $\mathrm{P}$ & & \\
\hline \multicolumn{7}{|l|}{ OFIDIOS } \\
\hline Boa constrictor occidentalis & A & & & & LR & \\
\hline Boiruna maculata & NA & & & & & $\mathrm{P}$ \\
\hline Bothrops jararacussu & A & & & & & \\
\hline Chironius bicarinatus & V & & & & & MP \\
\hline Clelia rustica & NA & & & & & $\mathrm{P}$ \\
\hline Crotalus durissus terrificus & NA & & & & & MP \\
\hline Echinanthera occipitalis & NA & & & & & $\mathrm{P}$ \\
\hline Echinanthera poecilopogon & IC & & & & & MP \\
\hline Eunectes notaeus & $\mathrm{V}$ & & & & DD & MP \\
\hline Helicops infrataeniatus & NA & & & & & $\mathrm{P}$ \\
\hline Leptophis ahaetulla marginatus & NA & & & & & $\mathrm{P}$ \\
\hline Leptotyphlops munoai & NA & & & & & $\mathrm{P}$ \\
\hline Liophis almadensis & NA & & & & & $\mathrm{P}$ \\
\hline Liophis flavifrenatus & NA & & & & & $\mathrm{P}$ \\
\hline Liotyphlops ternetzii & NA & & & & & MP \\
\hline Lystrophis histricus & IC & & & & & MP \\
\hline Micrurus altirostris & NA & & & & & $\mathrm{P}$ \\
\hline Phalotris bilineatus & NA & & & & & $\mathrm{P}$ \\
\hline Philodryas olfersii olfersii & NA & & & & & $\mathrm{P}$ \\
\hline Pseudablabes agassizii & NA & & & & & MP \\
\hline Sibynomorphus turgidus & NA & & & & & MP \\
\hline Tachymenis chilensis & NA & $\mathrm{V}$ & V & V & & \\
\hline Tachymenis peruviana & NA & & $\mathrm{R}$ & $\mathrm{R}$ & & \\
\hline Tantilla melanocephala & NA & & & & & $\mathrm{P}$ \\
\hline Thamnodynastes strigatus & NA & & & & & $\mathrm{P}$ \\
\hline
\end{tabular}




\section{DISCUSION}

La gran proporción de taxa categorizados como Insuficientemente conocidos, es consecuencia en gran parte del descubrimiento de nuevas especies y subespecies. La categoría Insuficientemente conocida, recientemente incorporada a la legislación, revela el nivel de desconocimiento sobre la fauna silvestre, mientras que el método utilizado para la evaluación permite la detección de los aspectos sobre los que falta información. Según se afirma en el Capítulo 7 de la publicación de Lavilla et al. (2000), esta explicitación del desconocimiento conduce a una "situación de privilegio a la hora de establecer nuevas líneas de investigación y priorizar proyectos de estudio, los que deberían focalizarse en los taxa que han sido categorizados como Insuficientemente conocidos". Sin embargo, hasta que esto no ocurra, es motivo de preocupación la alta proporción de taxa ubicados en esta categoría. La carencia de información que imposibilita determinar su estado de conservación constituye también un factor de amenaza, dado que no permite tomar decisiones adecuadas para su manejo.

También es motivo de alerta el hecho de que muchos taxa que fueron asignados a la categoría Insuficientemente conocida presenten aspectos negativos para su sobrevivencia, tales como distribuciones restringidas, acciones extractivas o poca protección. Como lo señalaron Lavilla \& Barrionuevo (2001), merecen especial atención porque entre ellos puede haber formas Vulnerables, Amenazadas o en Peligro de Extinción.

La mayoría de la herpetofauna cuya conservación reviste algún grado de amenaza tiene áreas de distribución pequeñas en Argentina. Esto adquiere mayor relevancia si se tiene en cuenta además que muchas especies no están contenidas en unidades de conservación o están pobremente protegidas. Los anfibios son el grupo que más acusa una distribución geográfica restringida, a lo cual se suma que dentro del área de su distribución cada especie de anfibio se encuentra en sitios muy específicos.

$\mathrm{Al}$ igual que los anfibios, todas las tortugas y la mayoría de los saurios y ofidios tienen poca plasticidad en el uso del hábitat. La vulnerabilidad inherente resultante de esta condición, se ve agravada con la alteración o destrucción del hábitat. Esto debe tenerse en cuenta a la hora de establecer políticas de conservación.

Una parte importante de la herpetofauna cuya conservación reviste algún grado de amenaza, está afectada por acciones antrópicas, en especial los reptiles. El comercio de algunas especies alcanzó altos niveles, lo cual determinó su inclusión en los Apéndices I y II de CITES. Es el caso de las tortugas marinas Caretta caretta, Chelonia mydas y Dermochelys coriacea, las tortugas terrestres de la familia Testudinidae como Chelonoidis chilensis, C. carbonaria y C. donosobarrosi, los yacarés Caiman latirostris y C. yacare, y las boasBoa constrictor occidentalis, Epichrates cenchria alvarezi, E. c. crassus y Eunectes notaeus.

Según la escala utilizada para ponderar el tamaño, las especies más afectadas por las acciones extractivas son las de tamaño mediano (dentro de lo que "mediano" puede significar en el contexto de los tamaños del grupo en cuestión). Las causales de extracción de herpetozoos parecen no estar ligadas al tamaño como ha sido mencionado para algunos mamíferos (Cofré \& Marquet 1999).

Las tortugas son uno de los grupos de reptiles tradicionalmente más comercializados para su uso como mascota o más explotado por las economías de subsistencia regionales (Richard et al. en Lavilla et al. 2000). Entre éstas, Chelonoidis chilensis es el reptil argentino sobre el que se ejerce mayor presión de caza extractiva multipropósito (Richard et al. op. cit.).

Todos los ofidios generan en el hombre distintos tipos de reacción que resultan en la extracción de individuos de las poblaciones naturales (Scrocchi et al. en Lavilla et al. 2000). Son perseguidos por temor, odio o repulsión, mientras que algunas especies son capturadas para ser utilizadas como mascotas, para extracción de venenos, o son comercializadas por su cuero. Algunos de los ofidios más amenazados por acciones extractivas tienen distribuciones restringidas, poca plasticidad en el uso del hábitat y/o bajas abundancias relativas.

Las dos especies de yacarés, explotadas intensivamente en el pasado reciente (hasta 1990), actualmente gozan de medidas de control a nivel nacional e internacional y sólo subsiste una reducida explotación relacionada con el consumo de su carne por parte de algunas comunidades locales (Waller et al. en Lavilla et al. 2000).

La utilización de algunas especies de saurios 
en el comercio de mascotas se ha incrementado recientemente (Avila et al. en Lavilla et al. 2000), aunque la alteración del hábitat en todas sus variantes parece ser la amenaza más extendida.

La mayoría de los anfibios presentan bajos niveles de acciones extractivas, principalmente ocasionadas por temor, repulsión o por ser utilizados con fines curativos o místicos por ciertas culturas. Muy pocos anfibios sufren una extracción considerable, debido al valor que alcanzan como mascotas o por su uso como animales experimentales o para alimento. Entre éstos merecen mencionarse Leptodactylus laticeps y Melanophryniscus s. stelzneri. El mayor impacto sobre los anfibios reconocido por los especialistas que trabajaron en la recalificación, es el ejercido por la pérdida irreversible del hábitat, que tuvo consecuencias graves en los casos de Atelognathus patagonicus y Telmatobius atacamensis. También merece destacarse la acción negativa que ejercen sobre los anfibios de montaña del noroeste argentino, algunas actividades relacionadas al turismo de aventura (Lavilla 2000). Paradójicamente, este tipo de turismo es aceptado por el común de la gente como indicador de una actitud positiva hacia la naturaleza.

En relación al grado de protección, el grupo de las tortugas es el menos favorecido en Argentina, en particular las tortugas marinas, dado que no existen formas legales para protegerlas en el país pues sus áreas de nidificación se encuentran fuera del territorio.

Tanto el método utilizado para evaluar el estado de conservación, como el análisis particular realizado en el presente trabajo, han permitido detectar las amenazas de origen antrópico que actuaron y continúan actuando sobre los herpetozoos. Esta identificación es de suma utilidad para encaminar correctamente medidas de protección para las especies. En un estudio sobre las especies de Estados Unidos que cambiaron de estatus de conservación (Abbitt \& Scott 2001), se encontraron diferencias entre las especies recuperadas o en recuperación y aquellas en declinación. Las primeras habían estado sometidas a contaminación, turismo de aventura, sobreexplotación y/o colecta, mientras que las segundas habían sido afectadas por la construcción de represas, drenajes, apertura de canales, depredadores exóticos y obras de desarrollo. La diferencia más grande entre estos dos conjuntos de amenazas es la facilidad con la que el daño que causan puede ser reparado. Los efectos del primer conjunto de amenazas son claros, los métodos de control son directos y las especies suelen responder a éstos rápidamente. Por el contrario, los efectos de las represas, los drenajes, las canalizaciones y las especies exóticas depredadoras, son mucho más complejos, virtualmente permanentes y se extienden a través de múltiples jurisdicciones políticas y categorías de propiedad. El efecto de las especies exóticas depredadoras, que ha sido identificado en Estados Unidos como la segunda amenaza para las especies nativas, es particularmente difícil de re-solver, dado que una vez establecidas su remoción probablemente nunca pueda lograrse de manera completa.

La comparación de la nueva categorización con la calificación pre-existente, revela la importancia de la actualización a través de métodos objetivos, que hacen al proceso repetible y verificable y cuya aplicación al conjunto garantiza una evaluación estandarizada. También se destaca la importancia de efectuar la evaluación mediante talleres de especialistas. Si bien para aplicar el método de Reca et al. (1994) no se requiere ser especialista si se dispone de la información sobre las especies, para la interpretación de los resultados y la asignación de las categorías, la discusión entre expertos es enriquecedora e incrementa la certeza en la categorización.

Es evidente la necesidad de actualizar las categorizaciones periódicamente, con el objeto de que los administradores de la fauna tengan una herramienta útil que refleje lo más fielmente posible la situación de las especies en el país, situación dinámica y cambiante que resulta de las modificaciones de los hábitats, de las presiones directas sobre las poblaciones naturales y de la protección que sobre ellas se ejerce.

En cuanto a la herpetofauna en particular, su conocimiento en Argentina ha experimentado un avance notorio en las últimas dos décadas, con una alta tasa de descubrimiento de nuevos taxa e incorporación de otros al elenco faunístico del país. Es necesario que este avance en el conocimiento biológico y taxonómico vaya acompañado a nivel gubernamental y no gubernamental por acciones que aseguren su conservación.

No pueden efectuarse interpretaciones com- 
parativas acerca del estatus de conservación de las especies en los distintos países considerados, debido a que las categorizaciones fueron realizadas en tiempos distintos y con métodos diferentes. Además, las categorías responden a definiciones no equivalentes. Para la obtención de resultados comparables es necesario adoptar el mismo método, tal como fue señalado por especialistas de varios países de Sudamérica (Grigera 2000) y por Grigera \& Ubeda (2002) para el caso particular de Argentina. Un diagnóstico objetivo de la situación de las especies en su área de distribución, inde-pendiente de sus límites políticos, constituye una herramienta indispensable para planificar y ejecutar políticas de conservación efectivas, tanto nacionales como conjuntas entre los países.

\section{AGRADECIMIENTOS}

Este trabajo fue posible gracias a la tarea de los especialistas que aportaron sus conocimientos y esfuerzo para efectuar la recategorización de la herpetofauna de Argentina.

\section{BIBLIOGRAFIA}

Аввітт, R.J.F. \& J.M. Sсотт. 2001. Examining differences between recovered and declining endangered species. Conserv. Biol. 15(5):12741284.

Avila, L.J. \& J.C. Acosta. 1996. Evaluación del estado de conservación de la fauna de saurios anfisbénidos y anfibios de la provincia de Córdoba (Argentina). FACENA 12:77-92.

Bertonatti, C. 1994. Lista propuesta de anfibios y reptiles amenazados de extinción. Cuad. herpetol. 8(1):164-171.

Bertonatti, C. 2000. Nuestro Libro Rojo. Vida Silvestre, Fundación Vida Silvestre Argentina 73:21.

Bertonatti, C. 2001. Nuestro Libro Rojo. Vida Silvestre, Fundación Vida Silvestre Argentina 78:51.

Bertonatti, C. \& J. Corcuera (eds.). 2001. Situación Ambiental Argentina 2000. Fundación Vida Silvestre Argentina. Buenos Aires, 440 pp.

Bertonatti, C. \& F. González. 1992. Lista de Vertebrados Argentinos amenazados de extinción. Fundación Vida Silvestre Argentina. Boletín Técnico 8, 33 pp.

Bertonatti, C. \& R. Banchs. 1993. La represa Yacyretá y su impacto ambiental. Diagnóstico y recomendaciones. Fundación Vida Silvestre Argentina. Boletín Técnico 18, 49 pp.

Cabrera, M.R. 1996. Lista y distribución geográfica de saurios, anfisbenas y tortugas (Amniota, Rep- tilia) de la Provincia de Córdoba: 215-238. In: I.E. di Tada \& E.H. Bucher (eds.), Biodiversidad de la Provincia de Córdoba, Volumen 1, Fauna. Universidad Nacional de Río Cuarto. Río Cuarto, Argentina, 373 pp.

Cabrera, M.R. 1998. Las tortugas continentales de Sudamérica austral. Córdoba, Argentina. 108 pp +6 lam.

Centro Editor de América Latina S.A. 1983-1986. Fauna Argentina, Buenos Aires. Fascículos $\mathrm{N}^{\circ}$ $3,14,35,56,60,64$ y 103 .

Chani, J., C. Borghi \& M. Brasesco. 1989. Fauna silvestre de la Provincia de Río Negro. Una evaluación: 385-404. In: Actas Primeras Jornadas Nacionales de Fauna Silvestre. Universidad Nacional de La Pampa y Gobierno de La Pampa (eds.). Santa Rosa, La Pampa, 671 pp.

ChÉBez, J.C. 1994. Los que se van. Especies argentinas en peligro. Ed. Albatros. Buenos Aires, 604 pp.

Christie, M. 1995a. Anfibios patagónicos: 12-18. In: C. Ubeda \& D. Grigera (eds.), Recalificación del Estado de Conservación de la Fauna Silvestre Argentina. Región Patagónica. Secretaría de Recursos Naturales y Ambiente Humano \& Consejo Asesor Regional Patagónico de la Fauna Silvestre. Buenos Aires, 95 pp.

Christie, M. 1995b. Reptiles patagónicos: 19-28. In: C. Ubeda \& D. Grigera (eds.), Recalificación del Estado de Conservación de la Fauna Silvestre Argentina. Región Patagónica. Secretaría de Recursos Naturales y Ambiente Humano \& Consejo Asesor Regional Patagónico de la Fauna Silvestre. Buenos Aires, 95 pp.

Cofré, H. \& P.A. Marquet. 1999. Conservation status, rarity, and geographic priorities for conservation of Chilean mammals: an assessment. Biol. Conserv. 88:53-68.

Decreto No 691. 1981. Boletín Oficial de la República Argentina NE 24644, 7 de abril de 1981.

Decreto N ${ }^{\circ}$ 666. 1997. Boletín Oficial de la República Argentina NE 28695, 25 de julio de 1997.

Dirección de Parques Nacionales y Vida Silvestre FundaCión Moisés BerTonI. 1997. Fauna amenazada del Paraguay. Asunción, Paraguay, 77 pp.

Di Tada, I.E., M.V. ZavatTieri, M.E. Bridarolli, N.E. Salas \& A.L. Martino. 1996. Anfibios anuros de la Provincia de Córdoba: 191-213. In: I.E. di Tada \& E.H. Bucher (eds.), Biodiversidad de la Provincia de Córdoba, Volumen 1, Fauna. Universidad Nacional de Río Cuarto. Río Cuarto, Argentina, $373 \mathrm{pp}$.

Ergueta S., P. \& C. De Morales (eds.). 1996. Libro Rojo de los Vertebrados de Bolivia. Centro de Datos para la Conservación, La Paz, Bolivia, 347 pp.

FreiberG, M. 1970. El mundo de los Ofidios. Ed. Albatros. Buenos Aires, 134 pp.

Freiberg, M. 1979. El mundo de las tortugas. Ed. Albatros. Buenos Aires, 142 pp.

Gallardo, J.M. 1979. Importancia de la conservación 
de las faunas de anfibios y reptiles. Acta Zool. Lilloana 34:102-106.

Glade, A.A. (ED.). 1993. Libro Rojo de los vertebrados terrestres de Chile. 2da edición. Santiago, 65 pp.

Grigera, D. 2000. Criterios para la evaluación del estado de conservación de la fauna silvestre: 273-278. In: Cabrera, E., C. Mercolli \& R. Resquin (eds.), Manejo de Fauna Silvestre en Amazonia y Latinoamérica, Asunción, Paraguay, 578 pp.

Grigera, D. \& C. Ubeda. 2002. Una revisión de los trabajos sobre categorizaciones y prioridades de conservación de los vertebrados de Argentina. Ecología Austral 12:163-174.

Gruss, J.X. \& T. Waller. 1988. Diagnóstico y recomendaciones sobre la administración de recursos silvestres en Argentina: la década reciente (un análisis sobre la administración de la fauna terrestre). WWF, TRAFFIC Sudamérica, CITES. Buenos Aires, 113 pp.

LaVILla, E.O. 2000. El progreso y las ranas de alta montaña: 285-286. In: C. Bertonatti \& J. Corcuera (eds.), Situación Ambiental Argentina 2000. Fundación Vida Silvestre Argentina. Buenos Aires, 392 pp.

Lavilla, E.O. 2001. Amenazas, declinaciones poblacionales y extinciones en anfibios argentinos. Cuad. herpetol. 15(1):59-82.

LaVilla, E.O. \& J.S. Barrionuevo. 2001. ¿Cuál es la situación de los anfibios "Insuficientemente conocidos" en Argentina?: 53-54. In: Resúmenes IV Congreso Argentino de Herpetología. 18 al 22 de noviembre. Salta, Argentina, 92 pp.

Lavilla, E.O., E. Richard \& G.J. Scrocchi (EDS.). 2000. Categorización de los anfibios y reptiles de la República Argentina. Asociación Herpetológica Argentina. San Miguel de Tucumán, 97 pp.

LEY No 22421. 1981. Boletín Oficial de la República Argentina NE 24626, 12 de marzo de 1981.

Maneyro, R. \& J.A. Langone. 2001. Categorización de los anfibios del Uruguay. Cuad. herpetol. 15(2):107-118.

Morales Fagundes, S. \& S. Carreira Vidal. 2000. Calificación del estado de conservación de la fauna de ofidios (Reptilia, Serpentes) de Uruguay. FACENA 16:45-51.

NArosky, T. \& D. Yzurieta. 1987. Guía para la identificación de las aves de Argentina y Uruguay. Asociación Ornitológica del Plata. Vázquez Mazzini (eds.), Buenos Aires, 345 pp.
NúÑez, H., V. Maldonado \& R. Pérez. 1997. Reunión de trabajo con especialistas de herpetología para categorización de especies según estados de conservación. Noticiario Mensual Museo Nacional de Historia Natural 329:12-19.

Ortiz, J.C. 1988. Situación de la exportación de los vertebrados terrestres chilenos. Com. Mus. Reg. Concepción 2:37-41.

REATI, G.J. 1996. Serpientes de la provincia de Córdoba: 239-254. In: I.E. di Tada \& E.H. Bucher (eds.), Biodiversidad de la Provincia de Córdoba, Volumen 1, Fauna. Universidad Nacional de Río Cuarto. Río Cuarto, Argentina, 373 pp.

RecA, A., C. ÚbedA \& D. Grigera. 1994. Conservación de la fauna de tetrápodos. I. Un índice para su evaluación. Mastozoología Neotropical 1(1):17-28.

Reca, A.R., C. Ubeda \& D. Grigera (Coordinadores). 1996. Prioridades de Conservación de los Mamíferos de la Argentina. Mastozoología Neotropical 3(1):87-117.

Secretaría de Agricultura y Ganadería. 1983. Resolución 144/83, Anexo I, Boletín Oficial 8/ 4 y 2/5/83:35-65. Buenos Aires.

Serret, A. \& C. Bertonatti. 1994. Nunca más Yacyretá. Vida Silvestre, Fundación Vida Silvestre Argentina 36:10.

Servicio Agrícola y Ganadero. 1998. Cartilla de Caza. Departamento de Protección de los Recursos Naturales Renovables, Santiago, Chile, 84 pp.

Ubeda, C. \& D. Grigera (EDS.) 1995. Recalificación del Estado de Conservación de la Fauna Silvestre Argentina. Región Patagónica. Secretaría de Recursos Naturales y Ambiente Humano \& Consejo Asesor Regional Patagónico de la Fauna Silvestre. Buenos Aires, 95 pp.

Ubeda, C., C. Alonso \& M.S. Pillado. 1998. Alsodes gargola, un anfibio endémico patagónico con adaptaciones a la vida en altura: 1-9. In: Fauna Andinopatagónica: aportes a su conocimiento. Patagonia Silvestre, Serie Técnica No 2.

Ubeda, C, H. Zagarese, M. Díaz \& F. Pedrozo. 1999. First steps towards the conservation of the microendemic frog Atelognathus nitoi. Oryx 33(1):58-66.

YANOSKY, A.A. 1989. La ofidiofauna de la Reserva Ecológica El Bagual, Formosa: abundancia, utilización de los hábitats y estado de situación. Cuad. herpetol. 4(3):11-14. 
APENDICE. Especies y subespecies de herpetozoos de Argentina cuya conservación se encuentra amenazada. Categorización según Lavilla et al. (2000). EP= En Peligro de Extinción, $\mathrm{V}=$ Vulnerable, $\mathrm{A}=$ Amenazada. *= incluida en Apéndice I de CITES, **= incluida en Apéndice II de CITES, ***= incluidas en Apéndice II de CITES sólo poblaciones de Argentina.
ApPENDIX. Species and subspecies of herpetofauna in Argentina whose conservation is threatened. Categorization according to Lavilla et al. (2000). EP= In Danger of Extinction, $\mathrm{V}=$ Vulnerable, $\mathrm{A}=$ Threatened. $*=$ included in CITES Appendix I, **= included in CITES Appendix II, ***= included in CITES Appendix II, only populations in Argentina.

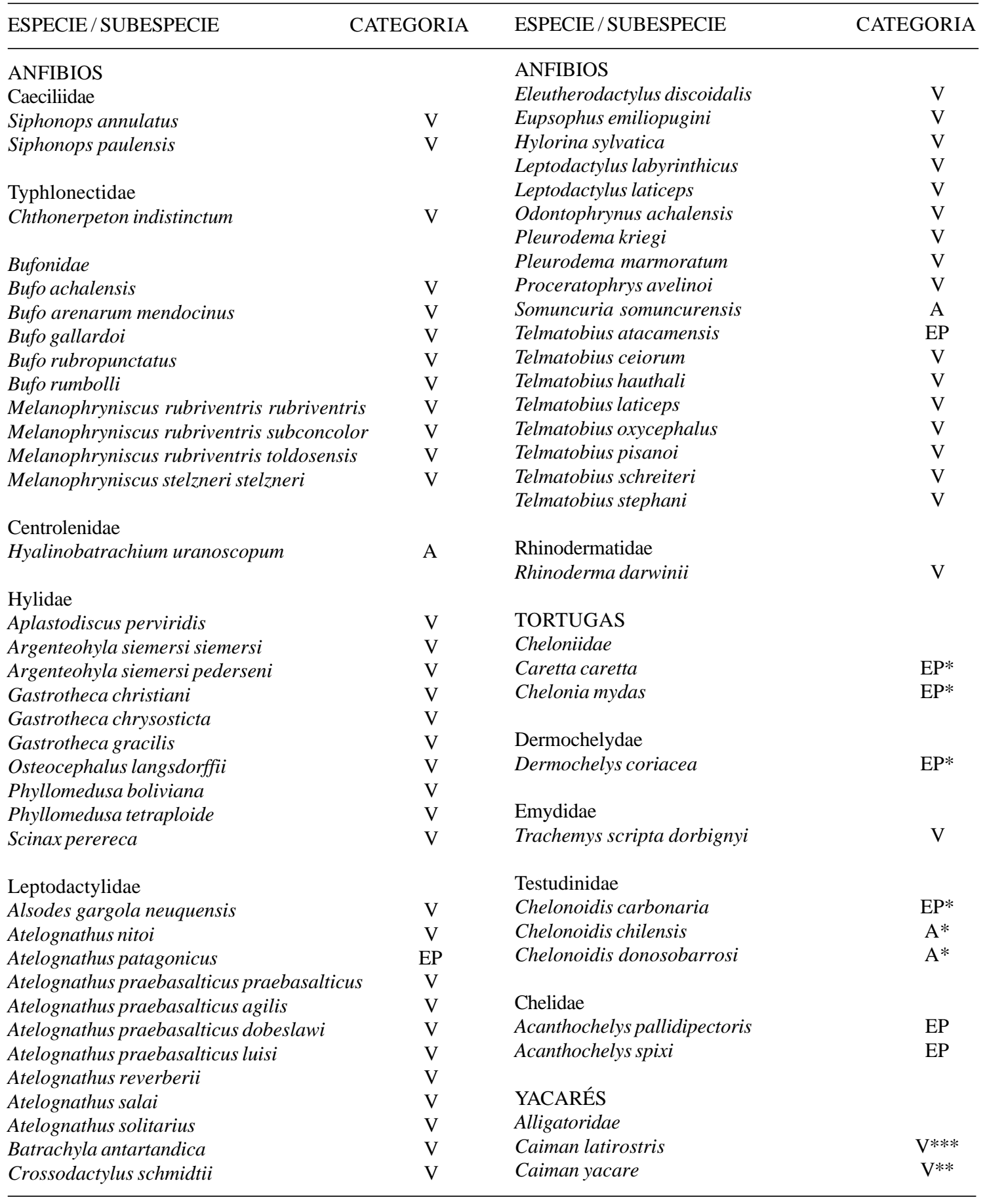


Gayana 67(1), 2003

Continuación Apéndice.

\begin{tabular}{|c|c|c|c|}
\hline ESPECIE / SUBESPECIE & CATEGORIA & ESPECIE / SUBESPECIE & CATEGORIA \\
\hline ANFIBIOS & & ANFIBIOS & \\
\hline SAURIOS & & Elapidae & \\
\hline Polychrotidae & & Micrurus lemniscatus & V \\
\hline Anisolepis grillii & A & & \\
\hline Anisolepis longicauda & A & Colubridae & \\
\hline Anisolepis undulatus & A & Atractus paraguayensis & V \\
\hline Diplolaemus leopardinus & V & Atractus reticulatus & $\mathrm{V}$ \\
\hline Leiosaurus paronae & $\mathrm{V}$ & Clelia plumbea & $\mathrm{V}$ \\
\hline Polychrus acutirostris & $\mathrm{V}$ & Chironius bicarinatus & $\mathrm{V}$ \\
\hline Pristidactylus achalensis & $\mathrm{V}$ & Chironius exoletus & V \\
\hline Pristidactylus casuhatiensis & $\mathrm{EP}$ & Dipsas indica bucephala & $\mathrm{V}$ \\
\hline \multirow[t]{2}{*}{ Urostrophus gallardoi } & A & Dipsas indica cisticeps & $\mathrm{V}$ \\
\hline & & Echinanthera cyanopleura & A \\
\hline Tropiduridae & & Erythrolamprus aesculapii venustissimus & $\mathrm{V}$ \\
\hline Liolaemus multimaculatus & $\mathrm{V}$ & Hydrops triangularis & $\mathrm{V}$ \\
\hline \multirow[t]{2}{*}{ Liolaemus rabinoi } & $\mathrm{EP}$ & Imantodes cenchoa & A \\
\hline & & Liophis elegantissimus & $\mathrm{V}$ \\
\hline Teiidae & & Liophis frenatus & $\mathrm{V}$ \\
\hline Cnemidophorus lacertoides & $\mathrm{V}$ & Liophis reginae macrosomus & V \\
\hline \multirow[t]{2}{*}{ Cnemidophorus serranus } & $\mathrm{V}$ & Liophis vanzolinii & V \\
\hline & & Oxyrhopus clathratus & V \\
\hline Amphisbaenidae & & Oxyrhopus petola & $\mathrm{V}$ \\
\hline \multirow{2}{*}{ Cercolophia borellii } & A & Phalotris reticulatus & $\mathrm{V}$ \\
\hline & & Pseudoboa haasi & $\mathrm{EP}$ \\
\hline OFIDIOS & & Pseudoeryx plicatilis plicatilis & A \\
\hline Leptotyphlopidae & & Rachidelus brazili & $\mathrm{V}$ \\
\hline Leptotyphlops albipunctus & $\mathrm{V}$ & Sibynomorphus mikani & V \\
\hline \multirow[t]{2}{*}{ Leptotyphlops vellardi } & $\mathrm{V}$ & Spilotes pullatus anomalepis & $\mathrm{V}$ \\
\hline & & Tomodon dorsatus & $\mathrm{V}$ \\
\hline Boidae & & Xenodon neuwiedii & V \\
\hline Boa constrictor occidentalis & $A^{*}$ & & \\
\hline Epicrates cenchria alvarezi & $\mathrm{V} * *$ & Viperidae & \\
\hline Epicrates cenchria crassus & $\mathrm{EP}^{* *}$ & Bothrops cotiara & $\mathrm{EP}$ \\
\hline \multirow[t]{2}{*}{ Eunectes notaeus } & $\mathrm{V} * *$ & Bothrops jararacussu & $\mathrm{A}$ \\
\hline & & Bothrops moojeni & $\mathrm{V}$ \\
\hline
\end{tabular}

Fecha de recepción: 19/08/02

Fecha de aceptación: 08/04/03 